\title{
$H^{2}$-boundedness of the pullback attractors for non-autonomous 2D-Navier-Stokes equations in bounded domains
}

\author{
Julia García-Luengo, Pedro Marín-Rubio, José Real \\ Dpto. de Ecuaciones Diferenciales y Análisis Numérico, Universidad de Sevilla, \\ Apdo. de Correos 1160, 41080 Sevilla, Spain
}

\begin{abstract}
We prove some regularity results for the pullback attractors of a non-autonomous 2D-Navier-Stokes model in a bounded domain $\Omega$ of $\mathbb{R}^{2}$. We establish a general result about $\left(H^{2}(\Omega)\right)^{2} \cap V$-boundedness of invariant sets for the associate evolution process. Then, as a consequence, we deduce that, under adequate assumptions, the pullback attractors of the non-autonomous 2D-Navier-Stokes equations are bounded not only in $V$ but also in $\left(H^{2}(\Omega)\right)^{2}$.
\end{abstract}

Key words: 2D-Navier-Stokes equations, pullback attractors, invariant sets, $H^{2}$-regularity.

Mathematics Subject Classifications (2010): 35B41, 35B65, 35Q30

ऋ Corresponding author: José Real

This work has been partially supported by Ministerio de Ciencia e Innovación (Spain) under project MTM2008-00088, and Junta de Andalucía grant P07-FQM02468. J.G.-L. is a fellow of Programa de FPU del Ministerio de Educación.

Email addresses: luengo@us.es (Julia García-Luengo), pmr@us.es

(Pedro Marín-Rubio), jreal@us.es (José Real). 


\section{Introduction and setting of the problem}

Let us consider the following problem for a non-autonomous 2D-Navier-Stokes system:

$$
\left\{\begin{array}{l}
\frac{\partial u}{\partial t}-\nu \triangle u+(u \cdot \nabla) u+\nabla p=f(t) \text { in } \Omega \times(\tau,+\infty), \\
\nabla \cdot u=0 \text { in } \Omega \times(\tau,+\infty), \\
u=0 \text { on } \partial \Omega \times(\tau,+\infty) \\
u(x, \tau)=u_{\tau}(x), \quad x \in \Omega
\end{array}\right.
$$

where $\Omega \subset \mathbb{R}^{2}$ is a bounded open set, with regular boundary $\partial \Omega$, the number $\nu>0$ is the kinematic viscosity, $u$ is the velocity field of the fluid, $p$ the pressure, $\tau \in \mathbb{R}$ is a given initial time, $u_{\tau}$ is the initial velocity field, and $f(t)$ a given external force field.

To set our problem in the abstract framework, we consider the following usual abstract spaces (see [1] and [2-4]):

$$
\mathcal{V}=\left\{u \in\left(C_{0}^{\infty}(\Omega)\right)^{2}: \operatorname{div} u=0\right\}
$$

$H=$ the closure of $\mathcal{V}$ in $\left(L^{2}(\Omega)\right)^{2}$ with inner product $(\cdot, \cdot)$ and associate norm $|\cdot|$, where for $u, v \in\left(L^{2}(\Omega)\right)^{2}$,

$$
(u, v)=\sum_{j=1}^{2} \int_{\Omega} u_{j}(x) v_{j}(x) \mathrm{d} x,
$$

$V=$ the closure of $\mathcal{V}$ in $\left(H_{0}^{1}(\Omega)\right)^{2}$ with scalar product $((\cdot, \cdot))$ and associate norm $\|\cdot\|$, where for $u, v \in\left(H_{0}^{1}(\Omega)\right)^{2}$,

$$
((u, v))=\sum_{i, j=1}^{2} \int_{\Omega} \frac{\partial u_{j}}{\partial x_{i}} \frac{\partial v_{j}}{\partial x_{i}} \mathrm{~d} x .
$$

We also consider the operator $A: V \rightarrow V^{\prime}$ defined by $\langle A u, v\rangle=((u, v))$. Denoting $D(A)=\left(H^{2}(\Omega)\right)^{2} \cap V$, then $A u=-P \Delta u, \forall u \in D(A)$, is the Stokes operator $\left(P\right.$ is the ortho-projector from $\left(L^{2}(\Omega)\right)^{2}$ onto $\left.H\right)$.

Now we define the continuous trilinear form $b$ on $V \times V \times V$ by

$$
b(u, v, w)=\sum_{i, j=1}^{2} \int_{\Omega} u_{i} \frac{\partial v_{j}}{\partial x_{i}} w_{j} \mathrm{~d} x, \quad \forall u, v, w \in V .
$$


It is well known that

$$
b(u, v, v)=0 \quad \text { for all } u, v \in V .
$$

We remember (see [2] or [3]) that there exists a constant $C_{1}>0$ only dependent on $\Omega$ such that

$$
\begin{gathered}
|b(u, v, w)| \leq C_{1}|u|^{1 / 2}\|u\|^{1 / 2}\|v\||w|^{1 / 2}\|w\|^{1 / 2}, \quad \forall u, v, w \in V, \\
|b(u, v, w)| \leq C_{1}|A u|\|v\||w|, \quad \forall u \in D(A), v \in V, w \in H,
\end{gathered}
$$

and

$$
|b(u, v, w)| \leq C_{1}|u|^{1 / 2}|A u|^{1 / 2} \| v|||w|, \quad \forall u \in D(A), v \in V, w \in H .
$$

Assume that $u_{\tau} \in H$ and $f \in L_{l o c}^{2}(\mathbb{R} ; H)$.

Definition 1.1 A solution of (1) is a function $u \in C([\tau, T] ; H) \cap L^{2}(\tau, T ; V)$ for all $T>\tau$, with $u(\tau)=u_{\tau}$, such that for all $v \in V$,

$$
\frac{d}{d t}(u(t), v)+\nu((u(t), v))+b(u(t), u(t), v)=(f(t), v)
$$

where the equation must be understood in the sense of $\mathcal{D}^{\prime}(\tau,+\infty)$.

Under the conditions above (e.g. cf. [2] or [3]), there exists a unique solution $u(\cdot)=u\left(\cdot ; \tau, u_{\tau}\right)$ of $(1)$. Moreover, this solution $u$ satisfies that $u \in C([\tau+$ $\varepsilon, T] ; V) \cap L^{2}\left(\tau+\varepsilon, T ;\left(H^{2}(\Omega)\right)^{2}\right)$ for every $\varepsilon>0$ and $T>\tau+\varepsilon$. In fact, if $u_{\tau} \in V$, then $u \in C([\tau, T] ; V) \cap L^{2}\left(\tau, T ;\left(H^{2}(\Omega)\right)^{2}\right)$ for every $T>\tau$.

Therefore, we can define a process $U=\{U(t, \tau), \tau \leq t\}$ in $H$ as

$$
U(t, \tau) u_{\tau}=u\left(t ; \tau, u_{\tau}\right) \quad \forall u_{\tau} \in H, \quad \forall \tau \leq t
$$

and the restriction of this process to $V$ is a process in $V$.

A pullback attractor for the process $U$ defined by (6) (cf. [5-7]) is a family $\hat{\mathcal{A}}=\{\mathcal{A}(t): t \in \mathbb{R}\}$ of compact subsets of $H$ such that

a) (invariance) $U(t, \tau) \mathcal{A}(\tau)=\mathcal{A}(t)$ for all $\tau \leq t$,

b) (pullback attraction) $\lim _{\tau \rightarrow-\infty} \sup _{u_{\tau} \in B} \inf _{v \in \mathcal{A}(t)}\left|U(t, \tau) u_{\tau}-v\right|=0$, for all $t \in \mathbb{R}$, for any bounded subset $B \subset H$. 
It can be proved (see [9]) that, under the above conditions, if in addition $f$ satisfies

$$
\int_{-\infty}^{0} e^{\mu r}|f(r)|^{2} d r<+\infty
$$

for some $0<\mu<2 \nu \lambda_{1}$, where $\lambda_{1}$ denotes the first eigenvalue of the Stokes operator $A$, then there exists a pullback attractor for the process $U$ defined by (6).

Several studies on this model have already been published (cf. [5], [8,9]). However, as far as we know, no one refers to the $H^{2}$-regularity we will consider in this paper.

In the next section we prove some results which, in particular, imply that, under suitable assumptions, any pullback attractor $\hat{\mathcal{A}}$ for $U$ satisfies that $\mathcal{A}(t)$ is a bounded subset of $\left(H^{2}(\Omega)\right)^{2} \cap V$, for every $t \in \mathbb{R}$ (for similar results for reaction-diffusion equations see [10], and for related results for Navier-Stokes equations see [11]).

\section{$2 \quad H^{2}$-boundedness of invariant sets}

In this section we prove that, under suitable assumptions, any family of bounded subsets of $H$ which is invariant for the process $U$, is in fact bounded in $\left(H^{2}(\Omega)\right)^{2} \cap V$.

First, we recall a result (cf. [2]) which will be used below.

Lemma 2.1 Let $X, Y$ be Banach spaces such that $X$ is reflexive, and the inclusion $X \subset Y$ is continuous. Assume that $\left\{u_{n}\right\}$ is a bounded sequence in $L^{\infty}\left(t_{0}, T ; X\right)$ such that $u_{n} \rightarrow u$ weakly in $L^{q}\left(t_{0}, T ; X\right)$ for some $q \in[1,+\infty)$ and $u \in C^{0}\left(\left[t_{0}, T\right] ; Y\right)$.

Then, $u(t) \in X$ and $\|u(t)\|_{X} \leq \liminf _{n \geq 1}\left\|u_{n}\right\|_{L^{\infty}\left(t_{0}, T ; X\right)}$, for all $t \in\left[t_{0}, T\right]$.

For each integer $n \geq 1$, we denote by $u_{n}(t)=u_{n}\left(t ; \tau, u_{\tau}\right)$ the Galerkin approximation of the solution $u\left(t ; \tau, u_{\tau}\right)$ of $(1)$, which is given by

$$
u_{n}(t)=\sum_{j=1}^{n} \gamma_{n j}(t) w_{j}
$$

and is the solution of

$$
\left\{\begin{array}{l}
\frac{d}{d t}\left(u_{n}(t), w_{j}\right)+\nu\left(\left(u_{n}(t), w_{j}\right)\right)+b\left(u_{n}(t), u_{n}(t), w_{j}\right)=\left(f(t), w_{j}\right), \\
\left(u_{n}(\tau), w_{j}\right)=\left(u_{\tau}, w_{j}\right) \quad j=1, \ldots, n
\end{array}\right.
$$


where $\left\{w_{j}: j \geq 1\right\} \subset V$ is the Hilbert basis of $H$ formed by the eigenvectors of the Stokes operator $A$. Observe that by the regularity of $\Omega$, all the $w_{j}$ belong to $\left(H^{2}(\Omega)\right)^{2}$.

We first prove the following result.

Proposition 2.2 Assume that $f \in L_{\text {loc }}^{2}(\mathbb{R} ; H)$. Then, for any bounded set $B \subset H$, any $\tau \in \mathbb{R}$, any $\varepsilon>0$ and any $t>\tau+\varepsilon$, the following three properties are satisfied:

i) The set $\left\{u_{n}\left(r ; \tau, u_{\tau}\right): r \in[\tau+\varepsilon, t], u_{\tau} \in B, n \geq 1\right\}$, is a bounded subset of $V$.

ii) The set of functions $\left\{u_{n}\left(\cdot ; \tau, u_{\tau}\right): u_{\tau} \in B, n \geq 1\right\}$, is a bounded subset of $L^{2}(\tau+\varepsilon, t ; D(A))$.

iii) The set of time derivatives functions $\left\{u_{n}^{\prime}\left(\cdot ; \tau, u_{\tau}\right): u_{\tau} \in B, n \geq 1\right\}$, is a bounded subset of $L^{2}(\tau+\varepsilon, t ; H)$.

\section{Proof.}

Let us fix a bounded set $B \subset H, \tau \in \mathbb{R}, \varepsilon>0, t>\tau+\varepsilon$, and $u_{\tau} \in B$.

Multiplying by $\gamma_{n j}(t)$ in (7), summing from $j=1$ to $n$, and using (2), we obtain

$$
\frac{1}{2} \frac{d}{d \theta}\left|u_{n}(\theta)\right|^{2}+\nu\left\|u_{n}(\theta)\right\|^{2}=\left(f(\theta), u_{n}(\theta)\right), \quad \text { a.e. } \theta>\tau .
$$

Observing that

$$
\begin{aligned}
\left|\left(f(\theta), u_{n}(\theta)\right)\right| & \leq \frac{1}{2 \nu \lambda_{1}}|f(\theta)|^{2}+\frac{\nu \lambda_{1}}{2}\left|u_{n}(\theta)\right|^{2} \\
& \leq \frac{1}{2 \nu \lambda_{1}}|f(\theta)|^{2}+\frac{\nu}{2}\left\|u_{n}(\theta)\right\|^{2}
\end{aligned}
$$

from (8) we deduce

$$
\frac{d}{d \theta}\left|u_{n}(\theta)\right|^{2}+\nu\left\|u_{n}(\theta)\right\|^{2} \leq \frac{1}{\nu \lambda_{1}}|f(\theta)|^{2},
$$

and integrating between $\tau$ and $r$,

$$
\begin{aligned}
\left|u_{n}(r)\right|^{2} & +\nu \int_{\tau}^{r}\left\|u_{n}(\theta)\right\|^{2} d \theta \\
& \leq\left|u_{\tau}\right|^{2}+\frac{1}{\nu \lambda_{1}} \int_{\tau}^{t}|f(\theta)|^{2} d \theta, \quad \forall r \in[\tau, t], \quad \forall n \geq 1 .
\end{aligned}
$$

Now, multiplying in (7) by $\lambda_{j} \gamma_{n j}(t)$, where $\lambda_{j}$ is the eigenvalue associated to the eigenvector $w_{j}$, and summing from $j=1$ to $n$, we obtain 


$$
\frac{1}{2} \frac{d}{d \theta}\left\|u_{n}(\theta)\right\|^{2}+\nu\left|A u_{n}(\theta)\right|^{2}+b\left(u_{n}(\theta), u_{n}(\theta), A u_{n}(\theta)\right)=\left(f(\theta), A u_{n}(\theta)\right)
$$

a.e. $\theta>\tau$. Observe that

$$
\left|\left(f(\theta), A u_{n}(\theta)\right)\right| \leq \frac{1}{\nu}|f(\theta)|^{2}+\frac{\nu}{4}\left|A u_{n}(\theta)\right|^{2},
$$

and by (5) and Young's inequality,

$$
\begin{aligned}
\left|b\left(u_{n}(\theta), u_{n}(\theta), A u_{n}(\theta)\right)\right| & \leq C_{1}\left|u_{n}(\theta)\right|^{1 / 2}\left\|u_{n}(\theta)\right\|\left|A u_{n}(\theta)\right|^{3 / 2} \\
& \leq \frac{\nu}{4}\left|A u_{n}(\theta)\right|^{2}+C^{(\nu)}\left|u_{n}(\theta)\right|^{2}\left\|u_{n}(\theta)\right\|^{4},
\end{aligned}
$$

where $C^{(\nu)}=27 C_{1}^{4}\left(4 \nu^{3}\right)^{-1}$.

Thus, from (10) we deduce

$$
\frac{d}{d \theta}\left\|u_{n}(\theta)\right\|^{2}+\nu\left|A u_{n}(\theta)\right|^{2} \leq \frac{2}{\nu}|f(\theta)|^{2}+2 C^{(\nu)}\left|u_{n}(\theta)\right|^{2}\left\|u_{n}(\theta)\right\|^{4},
$$

a.e. $\theta>\tau$.

From this inequality, in particular we deduce

$$
\begin{aligned}
\left\|u_{n}(r)\right\|^{2} & \leq\left\|u_{n}(s)\right\|^{2}+\frac{2}{\nu} \int_{\tau}^{t}|f(\theta)|^{2} d \theta \\
& +2 C^{(\nu)} \int_{s}^{r}\left|u_{n}(\theta)\right|^{2}\left\|u_{n}(\theta)\right\|^{4} d \theta
\end{aligned}
$$

for all $\tau \leq s \leq r \leq t$, and therefore, by Gronwall's lemma,

$$
\left\|u_{n}(r)\right\|^{2} \leq\left(\left\|u_{n}(s)\right\|^{2}+\frac{2}{\nu} \int_{\tau}^{t}|f(\theta)|^{2} d \theta\right) \exp \left(2 C^{(\nu)} \int_{\tau}^{t}\left|u_{n}(\theta)\right|^{2}\left\|u_{n}(\theta)\right\|^{2} d \theta\right)
$$

for all $\tau \leq s \leq r \leq t$.

Integrating this last inequality for $s$ between $\tau+\varepsilon / 2$ and $r$, we obtain

$$
\begin{aligned}
\left(r-\tau-\frac{\varepsilon}{2}\right)\left\|u_{n}(r)\right\|^{2} \leq & \left(\int_{\tau}^{t}\left\|u_{n}(s)\right\|^{2} d s+\frac{2(t-\tau)}{\nu} \int_{\tau}^{t}|f(\theta)|^{2} d \theta\right) \\
& \times \exp \left(2 C^{(\nu)} \int_{\tau}^{t}\left|u_{n}(\theta)\right|^{2}\left\|u_{n}(\theta)\right\|^{2} d \theta\right)
\end{aligned}
$$

for all $\tau+\varepsilon / 2 \leq r \leq t$, and in particular, 


$$
\begin{aligned}
\left\|u_{n}(r)\right\|^{2} \leq & \frac{2}{\varepsilon}\left(\int_{\tau}^{t}\left\|u_{n}(s)\right\|^{2} d s+\frac{2(t-\tau)}{\nu} \int_{\tau}^{t}|f(\theta)|^{2} d \theta\right) \\
& \times \exp \left(2 C^{(\nu)} \int_{\tau}^{t}\left|u_{n}(\theta)\right|^{2}\left\|u_{n}(\theta)\right\|^{2} d \theta\right)
\end{aligned}
$$

for all $\tau+\varepsilon \leq r \leq t$, for any $n \geq 1$.

From (9) and (13), the assertion in i) holds. Moreover, by (12),

$$
\begin{aligned}
\nu \int_{\tau+\varepsilon}^{t}\left|A u_{n}(\theta)\right|^{2} d \theta \leq & \left\|u_{n}(\tau+\varepsilon)\right\|^{2}+\frac{2}{\nu} \int_{\tau}^{t}|f(\theta)|^{2} d \theta \\
& +2 C^{(\nu)} \int_{\tau+\varepsilon}^{t}\left|u_{n}(\theta)\right|^{2}\left\|u_{n}(\theta)\right\|^{4} d \theta
\end{aligned}
$$

and therefore, by i), the assertion in ii) holds.

On the other hand, multiplying by the derivative $\gamma_{n j}^{\prime}(t)$ in (7), and summing from $j=1$ till $n$, we obtain

$$
\left|u_{n}^{\prime}(\theta)\right|^{2}+\frac{\nu}{2} \frac{d}{d \theta}\left\|u_{n}(\theta)\right\|^{2}+b\left(u_{n}(\theta), u_{n}(\theta), u_{n}^{\prime}(\theta)\right)=\left(f(\theta), u_{n}^{\prime}(\theta)\right),
$$

a.e. $\theta>\tau$.

Observing that

and by (4)

$$
\left|\left(f(\theta), u_{n}^{\prime}(\theta)\right)\right| \leq \frac{1}{4}\left|u_{n}^{\prime}(\theta)\right|^{2}+|f(\theta)|^{2}
$$

$$
\begin{aligned}
\left|b\left(u_{n}(\theta), u_{n}(\theta), u_{n}^{\prime}(\theta)\right)\right| & \leq C_{1}\left|A u_{n}(\theta)\right||| u_{n}(\theta) \|\left|u_{n}^{\prime}(\theta)\right| \\
& \leq \frac{1}{4}\left|u_{n}^{\prime}(\theta)\right|^{2}+C_{1}^{2}\left|A u_{n}(\theta)\right|^{2}\left\|u_{n}(\theta)\right\|^{2},
\end{aligned}
$$

we obtain from (14)

$$
\left|u_{n}^{\prime}(\theta)\right|^{2}+\nu \frac{d}{d \theta}\left\|u_{n}(\theta)\right\|^{2} \leq 2|f(\theta)|^{2}+2 C_{1}^{2}\left|A u_{n}(\theta)\right|^{2}\left\|u_{n}(\theta)\right\|^{2} .
$$

Integrating this last inequality, we deduce that

$$
\begin{aligned}
\int_{\tau+\varepsilon}^{t}\left|u_{n}^{\prime}(\theta)\right|^{2} d \theta \leq & \nu\left\|u_{n}(\tau+\varepsilon)\right\|^{2}+2 \int_{\tau}^{t}|f(\theta)|^{2} d \theta \\
& +2 C_{1}^{2} \sup _{\theta \in[\tau+\varepsilon, t]}\left\|u_{n}(\theta)\right\|^{2} \int_{\tau+\varepsilon}^{t}\left|A u_{n}(\theta)\right|^{2} d \theta
\end{aligned}
$$

and therefore iii) follows from i) and ii). 
Corollary 2.3 Assume that $f \in L_{\text {loc }}^{2}(\mathbb{R} ; H)$. Then, for any bounded set $B \subset$ $H$, any $\tau \in \mathbb{R}$, any $\varepsilon>0$, and any $t>\tau+\varepsilon$, the set $\bigcup_{r \in[\tau+\varepsilon, t]} U(r, \tau) B$ is a bounded subset of $V$.

Proof. This is a straightforward consequence of Lemma 2.1, assertion i) in Proposition 2.2, and the well known fact (e.g. cf. [1-4]) that for all $u_{\tau} \in$ $B$ the Galerkin approximations $u_{n}\left(\cdot ; \tau, u_{\tau}\right)$ converge weakly to $u\left(\cdot ; \tau, u_{\tau}\right)$ in $L^{2}(\tau, t ; V)$, and $u\left(\cdot ; \tau, u_{\tau}\right) \in C([\tau, t] ; H)$.

Assuming additional regularity for the time derivative of $f$, we can improve the above results.

Proposition 2.4 Assume that $f \in W_{\text {loc }}^{1,2}(\mathbb{R} ; H)$. Then, for any bounded set $B \subset H$, any $\tau \in \mathbb{R}$, any $\varepsilon>0$, and any $t>\tau+\varepsilon$, the following two properties are satisfied:

iv) The set of time derivatives $\left\{u_{n}^{\prime}\left(r ; \tau, u_{\tau}\right): r \in[\tau+\varepsilon, t], u_{\tau} \in B, n \geq 1\right\}$, is a bounded subset of $H$.

$v$ ) The set $\left\{u_{n}\left(r ; \tau, u_{\tau}\right): r \in[\tau+\varepsilon, t], u_{\tau} \in B, n \geq 1\right\}$ is a bounded subset of $D(A)$.

Proof. Let us fix a bounded set $B \subset H, \tau \in \mathbb{R}, \varepsilon>0, t>\tau+\varepsilon$, and $u_{\tau} \in B$.

As we are assuming that $f \in W_{\text {loc }}^{1,2}(\mathbb{R} ; H)$, we can differentiate with respect to time in (7), and then, multiplying by $\gamma_{n j}^{\prime}(t)$, and summing from $j=1$ to $n$, we obtain

$$
\frac{1}{2} \frac{d}{d \theta}\left|u_{n}^{\prime}(\theta)\right|^{2}+\nu\left\|u_{n}^{\prime}(\theta)\right\|^{2}+b\left(u_{n}^{\prime}(\theta), u_{n}(\theta), u_{n}^{\prime}(\theta)\right)=\left(f^{\prime}(\theta), u_{n}^{\prime}(\theta)\right)
$$

a.e. $\theta>\tau$.

From this inequality, taking into account that

$$
\left|\left(f^{\prime}(\theta), u_{n}^{\prime}(\theta)\right)\right| \leq \frac{\nu}{2}\left\|u_{n}^{\prime}(\theta)\right\|^{2}+\frac{1}{2 \nu \lambda_{1}}\left|f^{\prime}(\theta)\right|^{2},
$$

and by (3)

$$
\begin{aligned}
\left|b\left(u_{n}^{\prime}(\theta), u_{n}(\theta), u_{n}^{\prime}(\theta)\right)\right| & \leq C_{1}\left|u_{n}^{\prime}(\theta)\right|\left\|u_{n}^{\prime}(\theta)\right\|\left\|u_{n}(\theta)\right\| \\
& \leq \frac{\nu}{2}\left\|u_{n}^{\prime}(\theta)\right\|^{2}+\frac{C_{1}^{2}}{2 \nu}\left|u_{n}^{\prime}(\theta)\right|^{2}\left\|u_{n}(\theta)\right\|^{2},
\end{aligned}
$$

we deduce

$$
\frac{d}{d \theta}\left|u_{n}^{\prime}(\theta)\right|^{2} \leq \frac{1}{\nu \lambda_{1}}\left|f^{\prime}(\theta)\right|^{2}+\frac{C_{1}^{2}}{\nu}\left|u_{n}^{\prime}(\theta)\right|^{2}\left\|u_{n}(\theta)\right\|^{2}
$$


Integrating in the last inequality,

$$
\left|u_{n}^{\prime}(r)\right|^{2} \leq\left|u_{n}^{\prime}(s)\right|^{2}+\frac{1}{\nu \lambda_{1}} \int_{\tau}^{t}\left|f^{\prime}(\theta)\right|^{2} d \theta+\frac{C_{1}^{2}}{\nu} \int_{s}^{r}\left|u_{n}^{\prime}(\theta)\right|^{2}\left\|u_{n}(\theta)\right\|^{2} d \theta,
$$

for all $\tau \leq s \leq r \leq t$.

Thus, by Gronwall's inequality,

$$
\left|u_{n}^{\prime}(r)\right|^{2} \leq\left(\left|u_{n}^{\prime}(s)\right|^{2}+\frac{1}{\nu \lambda_{1}} \int_{\tau}^{t}\left|f^{\prime}(\theta)\right|^{2} d \theta\right) \exp \left(\frac{C_{1}^{2}}{\nu} \int_{\tau+\varepsilon / 2}^{t}\left\|u_{n}(\theta)\right\|^{2} d \theta\right),
$$

for all $\tau+\varepsilon / 2 \leq s \leq r \leq t$.

Now, integrating this inequality with respect to $s$ between $\tau+\varepsilon / 2$ and $r$, we obtain

$$
\begin{aligned}
(r-\tau-\varepsilon / 2)\left|u_{n}^{\prime}(r)\right|^{2} \leq & \left(\int_{\tau+\varepsilon / 2}^{t}\left|u_{n}^{\prime}(s)\right|^{2} d s+\frac{t-\tau}{\nu \lambda_{1}} \int_{\tau}^{t}\left|f^{\prime}(\theta)\right|^{2} d \theta\right) \\
& \times \exp \left(\frac{C_{1}^{2}}{\nu} \int_{\tau+\varepsilon / 2}^{t}\left\|u_{n}(\theta)\right\|^{2} d \theta\right)
\end{aligned}
$$

for all $\tau+\varepsilon / 2 \leq r \leq t$, and any $n \geq 1$. In particular, thus,

$$
\begin{aligned}
\left|u_{n}^{\prime}(r)\right|^{2} \leq & \frac{2}{\varepsilon}\left(\int_{\tau+\varepsilon / 2}^{t}\left|u_{n}^{\prime}(s)\right|^{2} d s+\frac{t-\tau}{\nu \lambda_{1}} \int_{\tau}^{t}\left|f^{\prime}(\theta)\right|^{2} d \theta\right) \\
& \times \exp \left(\frac{C_{1}^{2}}{\nu} \int_{\tau+\varepsilon / 2}^{t}\left\|u_{n}(\theta)\right\|^{2} d \theta\right)
\end{aligned}
$$

for all $\tau+\varepsilon \leq r \leq t$, and any $n \geq 1$.

From this inequality and properties i) and iii) in Proposition 2.2, we obtain iv).

On the other hand, multiplying again in (7) by $\lambda_{j} \gamma_{n j}(t)$, and summing once more from $j=1$ to $n$, we obtain

$$
\left(u_{n}^{\prime}(r), A u_{n}(r)\right)+\nu\left|A u_{n}(r)\right|^{2}+b\left(u_{n}(r), u_{n}(r), A u_{n}(r)\right)=\left(f(r), A u_{n}(r)\right),
$$

for all $r \geq \tau$. But

$$
\left|\left(u_{n}^{\prime}(r), A u_{n}(r)\right)\right| \leq \frac{2}{\nu}\left|u_{n}^{\prime}(r)\right|^{2}+\frac{\nu}{8}\left|A u_{n}(r)\right|^{2},
$$


and

$$
\left|\left(f(r), A u_{n}(r)\right)\right| \leq \frac{2}{\nu}|f(r)|^{2}+\frac{\nu}{8}\left|A u_{n}(r)\right|^{2}
$$

Therefore, taking into account (11), we deduce from (15) that

$$
\frac{\nu}{2}\left|A u_{n}(r)\right|^{2} \leq \frac{2}{\nu}\left(\left|u_{n}^{\prime}(r)\right|^{2}+|f(r)|^{2}\right)+C^{(\nu)}\left|u_{n}(r)\right|^{2}\left\|u_{n}(r)\right\|^{4}
$$

for all $r \geq \tau$.

Thus, since in particular $f \in C(\mathbb{R} ; H)$, from i) in Proposition 2.2, iv) and inequality (16), we deduce v).

As a direct consequence of the above, we can now establish our main results.

Theorem 2.5 Assume that $f \in W_{\text {loc }}^{1,2}(\mathbb{R} ; H)$. Then, for any bounded set $B \subset$ $H$, any $\tau \in \mathbb{R}$, any $\varepsilon>0$, and any $t>\tau+\varepsilon$, the set $\bigcup_{r \in[\tau+\varepsilon, t]} U(r, \tau) B$ is a bounded subset of $D(A)=\left(H^{2}(\Omega)\right)^{2} \cap V$.

Proof. This follows from Lemma 2.1, Proposition 2.4, and the well known facts that $u_{n}\left(\cdot ; \tau, u_{\tau}\right)$ converges weakly to $u\left(\cdot ; \tau, u_{\tau}\right)$ in $L^{2}(\tau, t ; V)$, and $u\left(\cdot ; \tau, u_{\tau}\right)$ belongs to $C([\tau+\varepsilon, t] ; V)$.

Theorem 2.6 Assume that $f \in L_{\text {loc }}^{2}(\mathbb{R} ; H)$, and $\hat{\mathcal{A}}=\{\mathcal{A}(t): t \in \mathbb{R}\}$ is a family of bounded subsets of $H$, such that $U(t, \tau) \mathcal{A}(\tau)=\mathcal{A}(t)$ for any $\tau \leq t$. Then:

i) For any $T_{1}<T_{2}$, the set $\bigcup_{t \in\left[T_{1}, T_{2}\right]} \mathcal{A}(t)$ is a bounded subset of $V$.

ii) If moreover $f^{\prime} \in L_{\text {loc }}^{2}(\mathbb{R} ; H)$, then for any $T_{1}<T_{2}$, the set $\bigcup_{t \in\left[T_{1}, T_{2}\right]} \mathcal{A}(t)$ is a bounded subset of $\left(H^{2}(\Omega)\right)^{2} \cap V$.

Proof. It is enough to observe that if $\tau<T_{1}-1$ is fixed, then

$$
\bigcup_{t \in\left[T_{1}, T_{2}\right]} \mathcal{A}(t) \subset \bigcup_{t \in\left[\tau+1, T_{2}\right]} U(t, \tau) \mathcal{A}(\tau)
$$

Now, apply Corollary 2.3 and Theorem 2.5. 
Acknowledgments

The authors would like to thank the referees for their careful reading on a previous version of the paper.

\section{References}

[1] J. L. Lions, Quelques méthodes de résolution des problèmes aux limites non linéaires, Dunod, Gauthier-Villars, Paris, 1969.

[2] J. C. Robinson, Infinite-dimensional dynamical systems, Cambridge University Press, 2001.

[3] R. Temam, Infinite Dimensional Dynamical Systems in Mechanics and Physics, Springer, New York, Second Edition, 1997.

[4] R. Temam, Navier-Stokes equations, Theory and Numerical Analysis, 2nd. ed., North Holland, Amsterdam, 1979.

[5] T. Caraballo, G. Łukaszewicz, and J. Real, Pullback attractors for asymptotically compact non-autonomous dynamical systems, Nonlinear Anal. 64 (2006), 484498.

[6] T. Caraballo, G. Łukaszewicz, and J. Real, Pullback attractors for nonautonomous 2D Navier-Stokes equations in unbounded domains, C. R. Math. Acad. Sci. Paris, 342 (2006), 263-268.

[7] P. E. Kloeden, Pullback attractors of nonautonomous semidynamical systems, Stoch. Dyn. 3 (2003), 101-112.

[8] P. Marín-Rubio and J. Real, On the relation between two different concepts of pullback attractors for non-autonomous dynamical systems, Nonlinear Anal. 71 (2009), 3956-3963.

[9] J. García-Luengo, P. Marín-Rubio, and J. Real, Pullback attractors in $V$ for non-autonomous $2 D$-Navier-Stokes equations and their tempered behaviour, submitted.

[10] M. Anguiano, T. Caraballo, and J. Real, $H^{2}$-boundedness of the pullback attractor for a non-autonomous reaction-diffusion equation, Nonlinear Anal. 72 (2010), 876-880.

[11] C. Guillopé, Comportement à l'infini des solutions des équations de NavierStokes et propriété des ensembles fonctionnels invariants (ou attracteurs), Ann. Inst. Fourier (Grenoble), 32 (1982), 1-37. 